\section{A novel method to determine hepatic segments using Sonazoid, an ultrasound contrast agent}

\author{
Taehyuk Ham 1 , Joo Hee Jeon', Younghoon Roh², Sungwook Lee ${ }^{3}$, Sangyoon Lee', \\ Heejin Kwon ${ }^{1}$, Jinhan Cho ${ }^{1}$
}

Departments of ${ }^{1}$ Radiology, ${ }^{2}$ Surgery, and ${ }^{3}$ Internal Medicine, Dong-A University Hospital, Dong-A University College of Medicine, Busan; ${ }^{4}$ Department of Radiology, Daedong Hospital, Busan, Korea

The conventional radiologic method for liver segmentation is based on the position of the hepatic and portal veins. However, during surgery, liver segments are resected based on the distribution of hepatic portal blood flow. This discrepancy can lead to a number of problems, such as miscommunication among clinicians, missing the location of the segment with the hepatic mass, and the risk of extended hepatic resection. We suggest a novel method to determine hepatic segments based on portal blood flow, as in the surgical approach, but by using high and low mechanical indexes in contrast-enhanced ultrasonography with Sonazoid. This approach is helpful for preoperatively determining hepatic segments and reducing the risk of missing the location of a hepatic tumor or extended hepatic resection.

Keywords: Ultrasonography; Contrast media; Liver; Sonazoid

\section{Introduction}

For intrahepatic malignant tumors such as hepatocellular carcinoma (HCC), intrahepatic cholangiocarcinoma (IHCC), or solitary liver metastasis, surgical removal is considered to be the firstline treatment option. As these tumors usually present with impaired liver function, surgeons seek to preserve the liver parenchyma during operations [1].

According to the method based on Couinaud's classification, the hepatic segment containing a tumor is determined via through a conventional radiologic examination based on the position of the hepatic and portal veins $[2,3]$. However, during surgery, portal-vein blood flow is used to locate the tumor; ligation of a specific branch of the hepatic portal vein is performed, and the liver segment is resected based on the distribution of the hepatic portal blood flow. This technique is termed the Glissonean pedicle approach $[4,5]$. The discrepancies between Couinaud's classification and the Glissonean pedicle approach can lead to a number of problems, such as miscommunication among clinicians, missing the location of the segment with the hepatic mass, and the risk of extended hepatic resection. Moreover, techniques to confirm the exact position of the tumor during the operation include indigo-carmine blue injection into the suspicious vessel or compressing the vessel

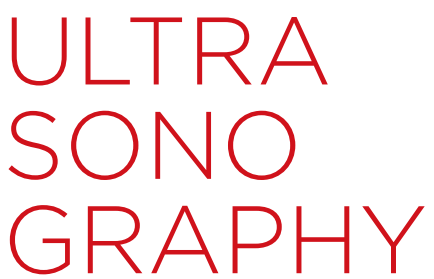

TECHNICAL NOTE

https://doi.org/10.14366/usg.19020 pISSN: 2288-5919 • elSSN: 2288-5943 Ultrasonography 2020;39:94-101

Received: April 9, 2019

Revised: July 17, 2019

Accepted: July 21, 2019

Correspondence to: Jinhan Cho, MD, PhD, Department of Radiology, Dong-A University Hospital, 26 Daesingongwon-ro, Seo-gu, Busan 49201, Korea

Tel. +82-51-240-5368

Fax. +82-51-240-3561

E-mail: jojini@dau.ac.kr

This is an Open Access article distributed under the terms of the Creative Commons Attribution NonCommercial License (http://creativecommons.org/ licenses/by-nc/4.0/) which permits unrestricted noncommercial use, distribution, and reproduction in any medium, provided the original work is properly cited.

Copyright $(2020$ Korean Society of Ultrasound in Medicine (KSUM)

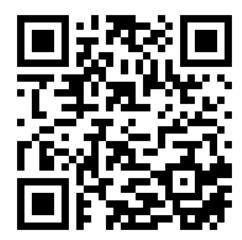

How to cite this article:

Ham T, Jeon JH, Roh Y, Lee S, Lee S, Kwon H, et al. A novel method to determine hepatic segments using Sonazoid, an ultrasound contrast agent. Ultrasonography. 2020 Jan; 39(1):94-101. 
with a finger for intraoperative ultrasonography (US). However, these techniques require significant skill and practice [6]. Therefore, development of a method to locate hepatic segments in the same way as the surgical method is needed.

US is a commonly used imaging modality. Contrast-enhanced ultrasonography (CEUS) has been available since development of US contrast agents and of the ability to visualize the unique signals of microbubbles in the vessels. Sonazoid (Daiichi Sankyo, Tokyo, Japan), a second-generation US contrast agent, consists of a lipidstabilized suspension of perflubutane microbubbles. CEUS with Sonazoid involves two phases of enhancement: the vascular phase and the late liver parenchyma-specific phase. This makes it a useful tool to diagnosis and assist in the treatment of hepatic tumors. The mechanical index (MI) is defined as the peak rarefactional pressure divided by the square root of the US frequency. It is related to the insonation power of the microbubbles within the US field. In response to US beams with a low MI $(<0.2)$, Sonazoid microbubbles remain static and scatter (Fig. 1A). Conventional CEUS with Sonazoid uses low-MI beams and helps to find hepatic tumors and tumor vessels. However, at a high $\mathrm{Ml}(>0.5)$, the Sonazoid microbubbles are destroyed (Fig. 1B). This technique shows promise for evaluating the enhancement patterns of focal liver lesions [7-10].

The purpose of this study was to examine whether the segment receiving blood from a particular portal vein can be differentiated with our novel technique, which uses both high- and low-MI CEUS with Sonazoid.

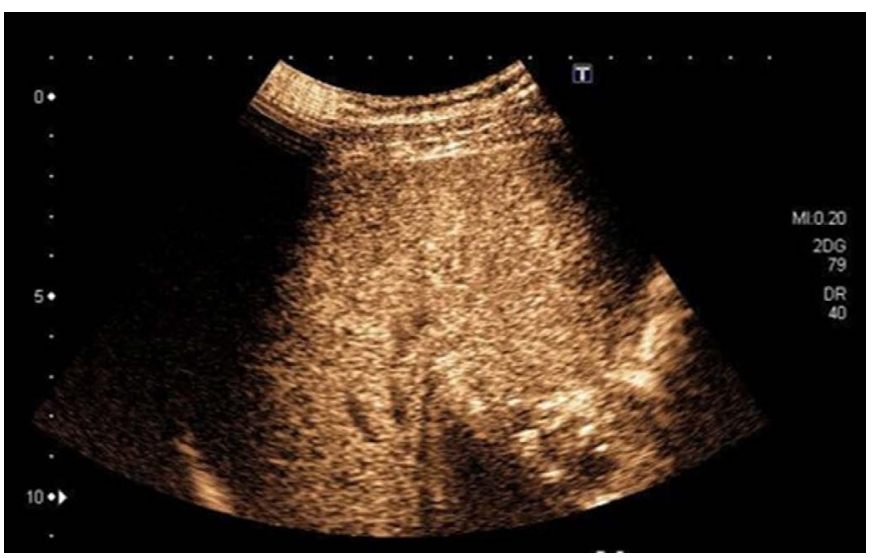

A

\section{Materials and Methods}

\section{Study Population}

Our institutional review board approved this retrospective study, and the requirement for informed consent was waived. From October 2016 to September 2018, patients with liver masses who underwent CEUS with Sonazoid were retrospectively evaluated. A total of 83 patients were reviewed. All patients had known hepatic tumors. In all cases, conventional CEUS with Sonazoid was performed to obtain a precise localization of the hepatic tumor before surgery. Among the 83 patients, 39 underwent additional CEUS with high and low $\mathrm{MI}$ for more accurate localization of masses considered to be near segment boundaries. One patient's data were excluded from the study because an adequate sonic view could not be obtained due to bowel invagination.

\section{US Protocol}

US was performed with an iU22 matrix ultrasound system (Philips, Seattle, WA, USA), with a 3-5 MHz convex transducer by two radiologists (one with more than 20 years of experience in abdominal radiology, the other with 10 years of experience in abdominal radiology).

First, conventional CEUS was performed to evaluate the liver tumors at a low Ml using Sonazoid (Fig. 2A). After 20 minutes, the remaining Sonazoid microbubbles were destroyed by performing high-MI US. Then, in patients with masses at the segment boundaries, an additional small dose $(0.006 \mathrm{~mL} / \mathrm{kg})$ of Sonazoid was injected. The radiologists targeted a specific portal vein with the narrowest field of view possible (Fig. 2B, C). To destroy the Sonazoid microbubbles, high-MI US was performed for more than 5 minutes

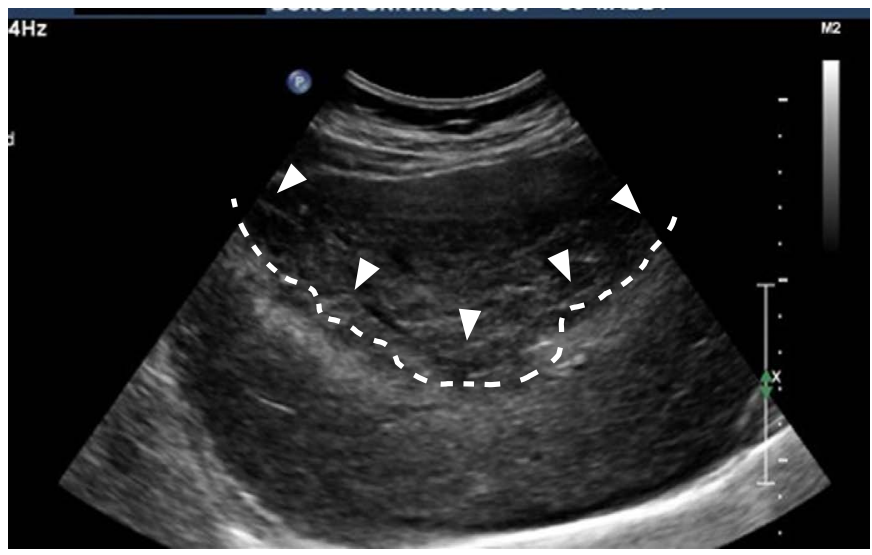

B

Fig. 1. Characteristics of Sonazoid according to the mechanical index (MI).

A. With a low MI $(<0.2)$, Sonazoid microbubbles are static and scatter in response to the ultrasound (US) beam. B. When the US beam has a high MI (>0.5), the Sonazoid microbubbles are destroyed. Arrowheads and dashes indicate disruption of the microbubbles. 
while targeting the specific portal vein. During these 5 minutes, the patient was asked to breathe as gently as possible. Finally, an additional low-MI CEUS evaluation was done to determine the hepatic segment with the tumor (Fig. 2B). A brief flow chart is shown in Fig. 3. The two radiologists determined the location of each tumor through consensus. All locations were determined without any controversy.

For a conventional CEUS examination, the recommended dose of Sonazoid is a bolus injection of $0.015 \mathrm{~mL} / \mathrm{kg}$ with saline flushing [7]. However, for the additional tests in this study, a smaller dose $(0.006 \mathrm{~mL} / \mathrm{kg})$ of Sonazoid without flushing was infused in patients with a high MI to induce sufficient destruction of the Sonazoid microbubbles. The additional small dose was determined in accordance with our previous study; we increased it from the minimum to a dose that showed clear contrast, but would not take too long to destroy.

\section{Analysis}

Sonograms of 38 patients were reviewed. Technical success of the US protocol was defined using the following grades: (1) excellent: segments show clear-cut boundaries, which can be easily distinguished; (2) good: segments are relatively easy to distinguish; (3) poor: segments show boundaries that are ill-defined, but still distinguishable; (4) indistinguishable: segments are not distinguishable (Fig. 4A-D).

Furthermore, the locations of tumors identified by our novel US

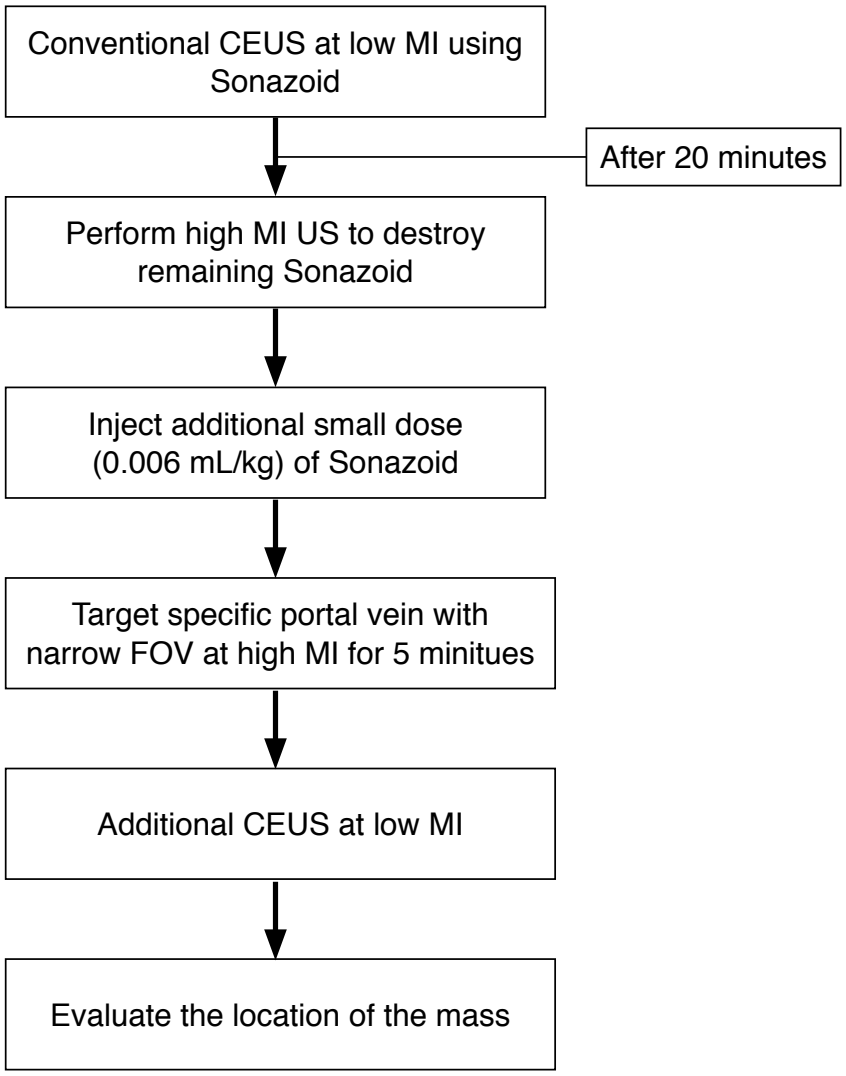

Fig. 3. Brief flow chart of the examination process. The flow chart shows the process of our novel ultrasonographic method. CEUS, contrast-enhanced ultrasonography; MI, mechanical index; US, ultrasonography; FOV, field of view.

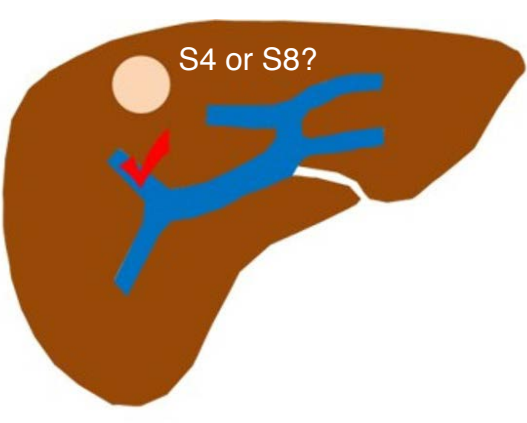

A

Fig. 2. Outline of a novel method to determine hepatic segments based on portal blood flow in contrast-enhanced ultrasonography with Sonazoid.

A. Conventional contrast-enhanced ultrasonographic evaluation of liver tumors is shown above. An intrahepatic tumor is identified, and the target portal vein is determined. B, C. Thereafter, an infusion of Sonazoid is injected and high-mechanical index (MI) ultrasonography scan of the target portal vein (S8) is performed. After destruction of the contrast agent in the specific portal vein (S8), a delayed ultrasonographic evaluation is performed with low MI to identify the hepatic segment. The tumor is visualized in $\mathrm{S} 4$ and found to be supplied by the $S 4$ portal vein (arrow) (B). A narrow field of view is used to destroy the contrast agent (Sonazoid) in the specific branch of the portal vein (C).

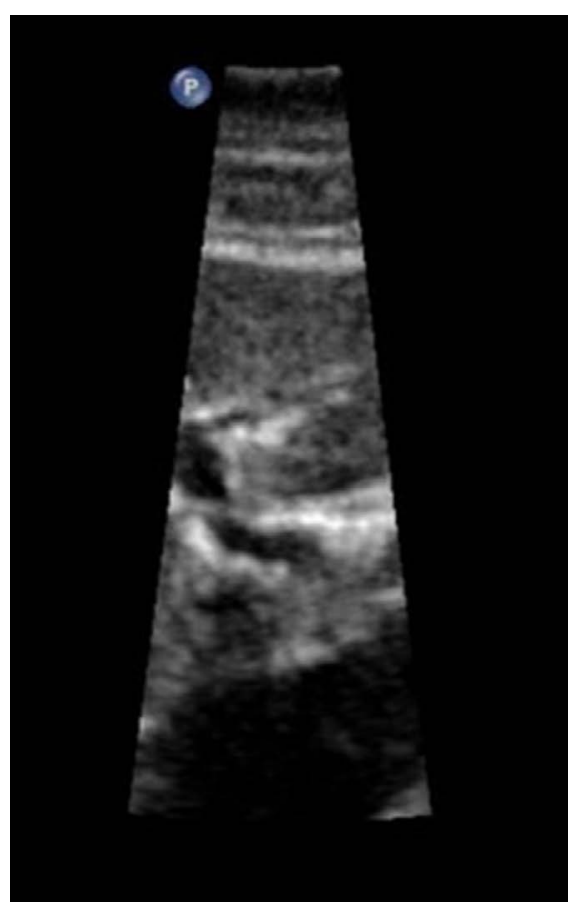

C 
method were compared with those identified by the conventional radiologic method.

The exact locations of the tumors were confirmed in 13 patients who underwent surgery with the Glissonean pedicle approach. We also compared the two US methods for these 13 patients.

\section{Results}

In total, 38 patients were included in our study. They were diagnosed with HCC $(n=21)$, metastasis $(n=11)$, intraductal papillary mucinous neoplasm of the bile duct $(n=2), \operatorname{IHCC}(n=1)$, angiomyolipoma $(n=1)$, focal nodular hyperplasia $(n=1)$, and hemangioma $(n=1)$. Thirty-four patients had only one mass. Four patients had two or more masses; three of these patients were diagnosed with metastasis, and one was diagnosed with HCC. In these four patients, conventional CEUS was performed for all masses, and our novel US method was performed on specific masses considered to be at the boundaries of the segment. Among the 38 patients, 35 underwent surgery, of whom 13 patients underwent surgery with the Glissonean pedicle approach, which

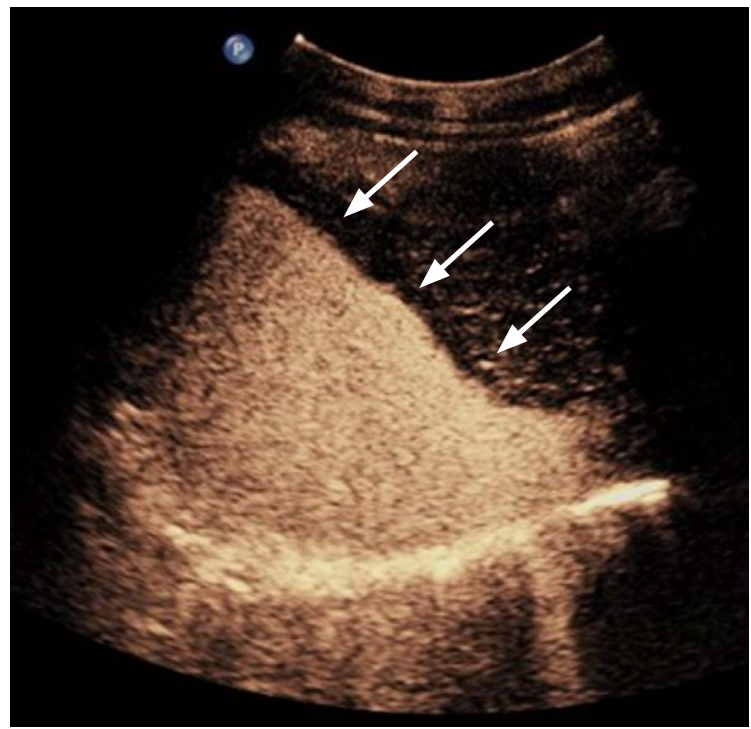

A

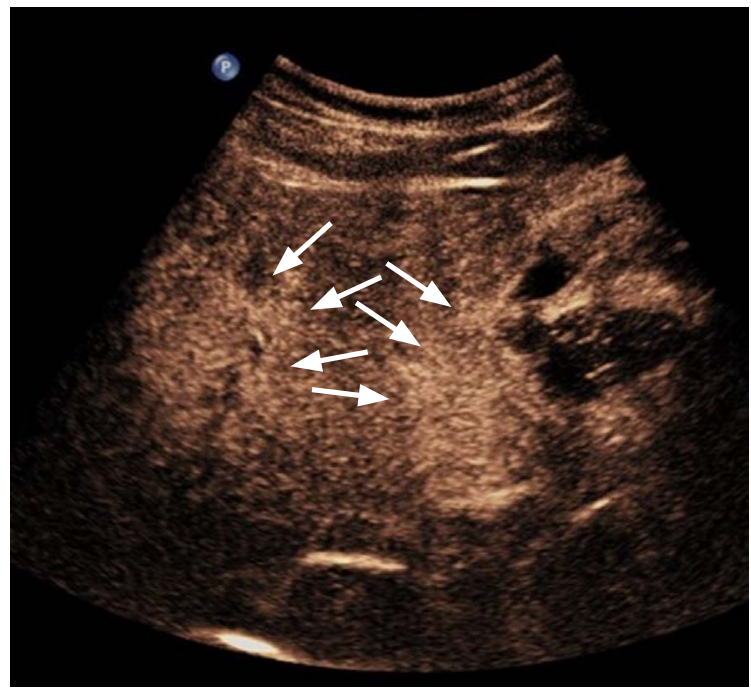

C

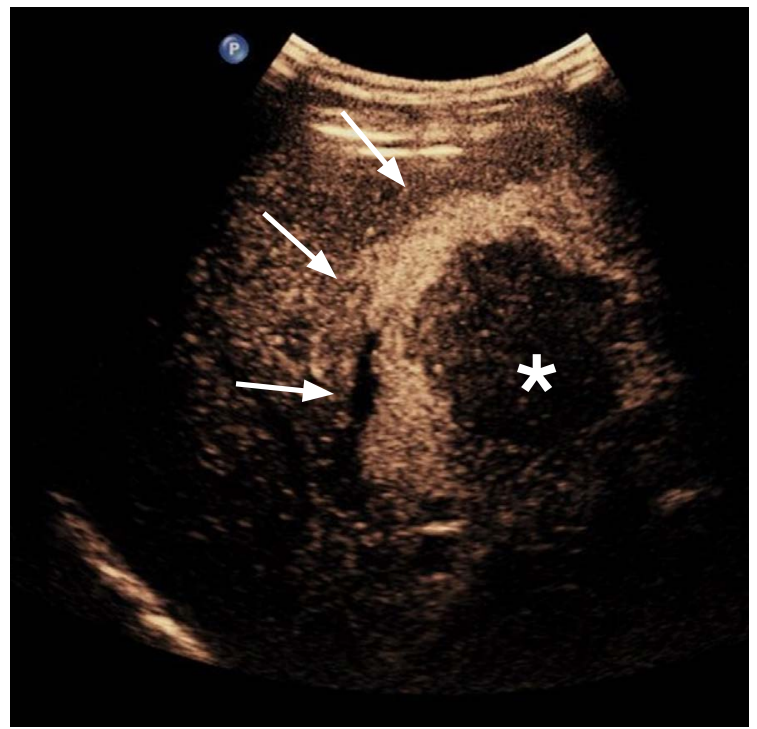

B

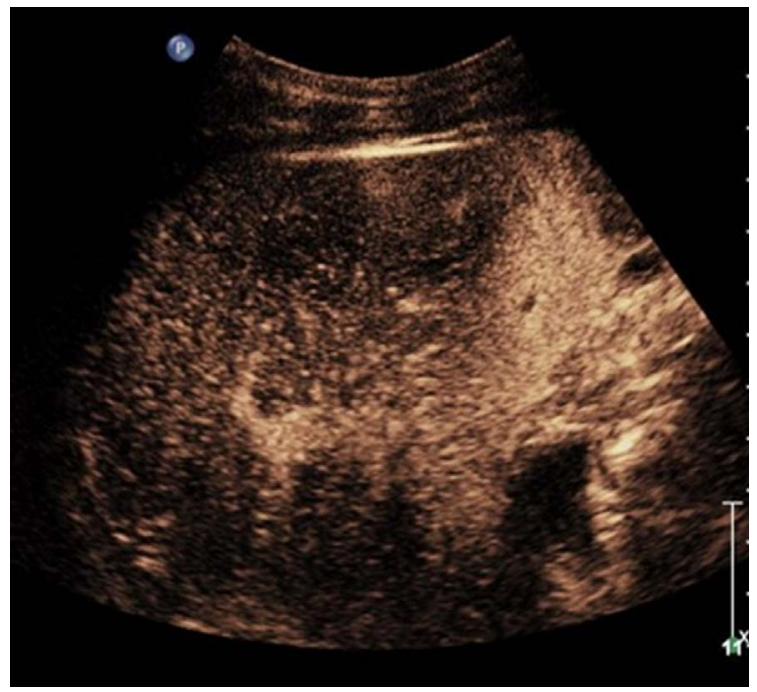

D

Fig. 4. Grades for evaluating technical success of the ultrasonographic procedure.

A. Excellent: Boundaries are clear-cut and segments are distinguishable (arrows, boundaries of the segments). B. Good: Segments are relatively easy to distinguish (star, hypoechoic mass; arrows, boundaries of the segments). C. Poor: Segment boundaries are ill-defined, but still distinguishable (arrows, boundaries of the segments). D. Indistinguishable: The segments are not distinguishable. 
Table 1. Characteristics of the 38 patients

\begin{tabular}{|c|c|}
\hline Characteristic & No. (\%) \\
\hline \multicolumn{2}{|l|}{ Age (yr) } \\
\hline Mean & 61.6 \\
\hline Median (range) & $62(43-78)$ \\
\hline \multicolumn{2}{|l|}{ Sex } \\
\hline Male & $27(71.1)$ \\
\hline Female & $11(28.9)$ \\
\hline Weight, mean \pm SD (kg) & $66.5 \pm 11.3$ \\
\hline Height, mean \pm SD (cm) & $162.3 \pm 8.8$ \\
\hline $\mathrm{BMI}$, mean $\pm \mathrm{SD}\left(\mathrm{kg} / \mathrm{m}^{2}\right)$ & $24.5 \pm 5.0$ \\
\hline \multicolumn{2}{|l|}{ Hepatic tumor } \\
\hline $\mathrm{HCC}$ & $21(55.3)$ \\
\hline Metastasis & $11(28.9)$ \\
\hline IPMN-B & $2(5.3)$ \\
\hline $\mathrm{IHCC}$ & $1(2.6)$ \\
\hline $\mathrm{FNH}$ & $1(2.6)$ \\
\hline Hemangioma & $1(2.6)$ \\
\hline AML & $1(2.6)$ \\
\hline \multicolumn{2}{|l|}{ Underlying disease } \\
\hline Liver cirrhosis & $21(55.3)$ \\
\hline Colorectal cancer & $10(26.3)$ \\
\hline Pancreatic cancer & $2(5.3)$ \\
\hline Chronic cholangitis & $2(5.3)$ \\
\hline Acute hepatopathy & $1(2.6)$ \\
\hline None & $2(5.3)$ \\
\hline \multicolumn{2}{|l|}{ Previous treatment } \\
\hline RFA & $3(7.9)$ \\
\hline TACE & $2(5.3)$ \\
\hline Radiation therapy & $2(5.3)$ \\
\hline \multicolumn{2}{|l|}{ Treatment } \\
\hline Wedge resection & $27(71.1)$ \\
\hline Left lateral sectionectomy & $5(13.2)$ \\
\hline Right hemihepatectomy & $1(2.6)$ \\
\hline TACE & $1(2.6)$ \\
\hline IORFA & $2(5.3)$ \\
\hline No further treatment & $2(5.3)$ \\
\hline Surgery with the Glissonean pedicle approacha) & $13(34.2)$ \\
\hline
\end{tabular}

SD, standard deviation; BMI, body mass index; HCC, hepatocellular carcinoma; IPMN-B, intraductal papillary mucinous neoplasm of the bile duct; IHCC, intrahepatic cholangiocarcinoma; $F N H$, focal nodular hyperplasia; $A M L$, angiomyolipoma; RFA, radio frequency ablation; TACE, transarterial chemoembolization; IORFA, intraoperative radiofrequency ablation.

${ }^{a}$ The exact segment of the tumor was confirmed in patients who underwent surgery with the Glissonean pedicle approach. This information is unavailable for the others.
Table 2. Technical success grades of the ultrasound protocol

\begin{tabular}{lr}
\hline \multicolumn{1}{c}{ Technical success } & No. (\%) \\
\hline Excellent & $10(26.3)$ \\
Good & $15(39.5)$ \\
Poor & $6(15.8)$ \\
Indistinguishable & $7(18.4)$ \\
Total & $38(100)$ \\
\hline
\end{tabular}

Excellent, boundaries are clear-cut and segments are distinguishable; Good, segments are distinguished relatively easily; Poor, segments show boundaries that are ill-defined, but still distinguishable; Indistinguishable, the segments are not distinguishable.

accurately demonstrated the location of the hepatic mass using the distribution of the portal vein blood flow.

Data from 38 patients were reviewed (Table 1), and the technical success of the new US protocol was determined for each case. The results were excellent in 10 cases (26\%), good in $15(40 \%)$, poor in six $(16 \%)$, and indistinguishable in seven (18\%). The sonograms of 25 patients showed boundaries that were easy or relatively easy to distinguish, and six showed faintly distinguishable segments. However, seven (18\%) showed indistinguishable segments (Table 2). Hence, the segment with the target mass could be localized in only 31 of the 38 patients (82\%) (Table 3).

Among the seven patients in whom the segment was indistinguishable, six patients had atrophic changes of the liver due to previous radiation therapy, chronic cholangitis, or advanced liver cirrhosis, and one patient had acute hepatopathy.

In the 25 patients who had distinguishable boundaries, the segment of the mass determined using the conventional radiologic method was compared with that determined using our novel method. In four of those 25 cases, it was more difficult to determine the segment of the mass via the conventional radiologic method, as the mass was located at a boundary. However, our novel US method helped determine the precise segment using portal-vein blood flow. Data from 13 patients who underwent surgery with the Glissonean pedicle approach were reviewed. The technical success grades of the US protocol for the 13 patients were as follows: 3 , excellent; 9, good, and 1, poor. The surgical location of the mass in all 13 patients was in concurrence with the segment preoperatively confirmed by our novel method. In two cases, the segment of the mass determined via the conventional radiologic method was inconsistent with that determined via our novel US method and surgically confirmed.

One of those cases showed a nodule in the $\$ 4$ segment based on Couinaud's classification on a computed tomography scan. However, our novel US method indicated that the location of the nodule was the $\$ 2$ segment. The S2 location was surgically confirmed, and a left 
Table 3. Hepatic segments of the target masses in the 31 patients

\begin{tabular}{|c|c|c|c|}
\hline Patient & $\begin{array}{l}\text { Conventional } \\
\text { segmentation }\end{array}$ & $\begin{array}{c}\text { Novel } \\
\text { segmentation }^{\mathrm{b}}\end{array}$ & $\begin{array}{l}\text { Glissonean pedicle } \\
\text { approach }^{(1)}\end{array}$ \\
\hline 1 & $\mathrm{~S} 2$ & $\mathrm{~S} 2$ & $\mathrm{~S} 2$ \\
\hline 2 & $S 2 / 3$ & $\$ 2 / 3$ & $S 2 / 3$ \\
\hline 3 & S3 & S3 & S3 \\
\hline 4 & $54 / 8$ & $54 / 8$ & $54 / 8$ \\
\hline 5 & S5 & S5 & S5 \\
\hline 6 & S5 & S5 & S5 \\
\hline 7 & S5 & S5 & S5 \\
\hline 8 & S5 & S5 & S5 \\
\hline 9 & S5 & S5 & S5 \\
\hline 10 & S6 & S6 & s6 \\
\hline 11 & 58 & S8 & 58 \\
\hline 12 & S4 & $\mathrm{S} 2$ & $\mathrm{~S} 2$ \\
\hline 13 & S4 & $\mathrm{S} 2$ & $\mathrm{~S} 2$ \\
\hline 14 & S2 & S2 & N/A \\
\hline 15 & $\mathrm{~S} 3$ and $\mathrm{S} 8$ & S3 and 58 & N/A \\
\hline 16 & $S 4 / 8$ & $54 / 8$ & N/A \\
\hline 17 & $S 4 / 8$ & $54 / 8$ & N/A \\
\hline 18 & $\mathrm{~S} 4$ and $\mathrm{S} 8$ & S4 and $\$ 8$ & N/A \\
\hline 19 & S5 & S5 & N/A \\
\hline 20 & $55 / 6$ & $55 / 6$ & N/A \\
\hline 21 & $\mathrm{~S} 5 / 8$ and $\mathrm{S} 6$ & $\mathrm{~S} 5 / 8$ and $\mathrm{S} 6$ & N/A \\
\hline 22 & $\mathrm{~S} 6$ and $\mathrm{S} 8$ & S6 and 58 & N/A \\
\hline 23 & S7 & S7 & N/A \\
\hline 24 & S7 & S7 & N/A \\
\hline 25 & S7 & S7 & N/A \\
\hline 26 & 58 & 58 & N/A \\
\hline 27 & S8 & S8 & N/A \\
\hline $28^{d)}$ & $s 2 / 3$ & S2 & N/A \\
\hline $29^{d)}$ & $52 / 54$ & S4 & N/A \\
\hline $30^{d)}$ & $54 / 8$ & 58 & N/A \\
\hline $31^{d)}$ & $57 / 8$ & S8 & N/A \\
\hline
\end{tabular}

N/A, not available.

${ }^{\text {a) }}$ Conventional radiologic method of segmentation, based on the position of the hepatic and portal veins. ${ }^{\text {b) }}$ Our novel method of segmentation using contrastenhanced ultrasonography, based on hepatic portal blood flow. ${ }^{\circ}$ The exact segment of the tumor was confirmed in patients who underwent surgery with the Glissonean pedicle approach. This information is unavailable for the others. ${ }^{d)}$ The four cases in which it was difficult to locate the accurate segment by conventional radiologic methods, but our novel method helped to determine the exact location. lateral sectionectomy was performed (Fig. 5A-D).

\section{Discussion}

CEUS has been used in echocardiography, vascular US, and abdominal US since 1996. However, it is often not used, although it has the advantage of being economical and involving less radiation than other modalities, such as computed tomography and magnetic resonance imaging. One reason why it was initially not used frequently is that first-generation contrast agents required a limited scan time because of the rapid breakdown of microbubbles. In the 2000s, the development of second-generation contrast agents and the technique of the low-MI mode in US machines promoted the use of CEUS [8]. SonoVue (Bracco, Milan, Italy) is another secondgeneration US contrast agent that is purely intravascular, without an interstitial extravascular phase. SonoVue can be seen only 3-5 minutes after injection. Sonazoid has a vascular phase and latephase contrast enhancement and can be seen up to 20 minutes after injection [9-11]. Due to the longer duration of contrast activity, Sonazoid was the more appropriate choice for the present study.

In our study, we introduced a novel method to determine hepatic segments using CEUS with Sonazoid. The target segment was distinguished in 31 of 38 patients ( $81 \%$ ). In the 25 cases in which the hepatic segment of the mass was identified by both methods, it might have been very helpful to reaffirm the location before surgery. In the four cases in which it was difficult to locate the accurate segment by conventional radiologic methods, our novel method helped determine the exact location. In particular, for patients who had discrepancies between the two methods, our novel method played an important role in determining the surgical site. We expect that our novel US method will help determine the exact scope of surgery and minimize resection of the liver. In all 13 patients who underwent surgery with the Glissonean pedicle approach, the segment determined by our novel US method was confirmed to be accurate. Although this study involved a small number of patients, the results suggest that this novel method works in a way similar to the Glissonean pedicle approach. Moreover, it is less invasive than surgical techniques such as indigo-carmine blue injection or finger compression. In order to confirm the hepatic segment by the direct vascular approach during surgery, additional dissection or puncture of the liver parenchyma may be needed to assess specific vessels. This is not only a difficult procedure, but may also cause other complications $[6,12]$. Moreover, if a precise plan can be formulated in advance, the range of resection during surgery is unlikely to change, and more accurate prediction of the remaining liver volume will be easier.

There are several limitations to this study. First, the actual 


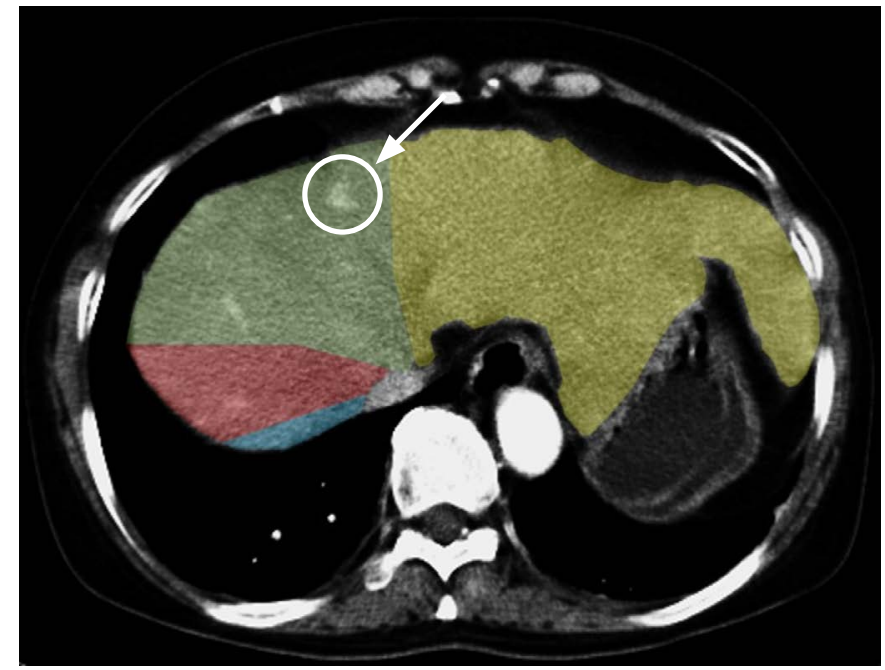

A

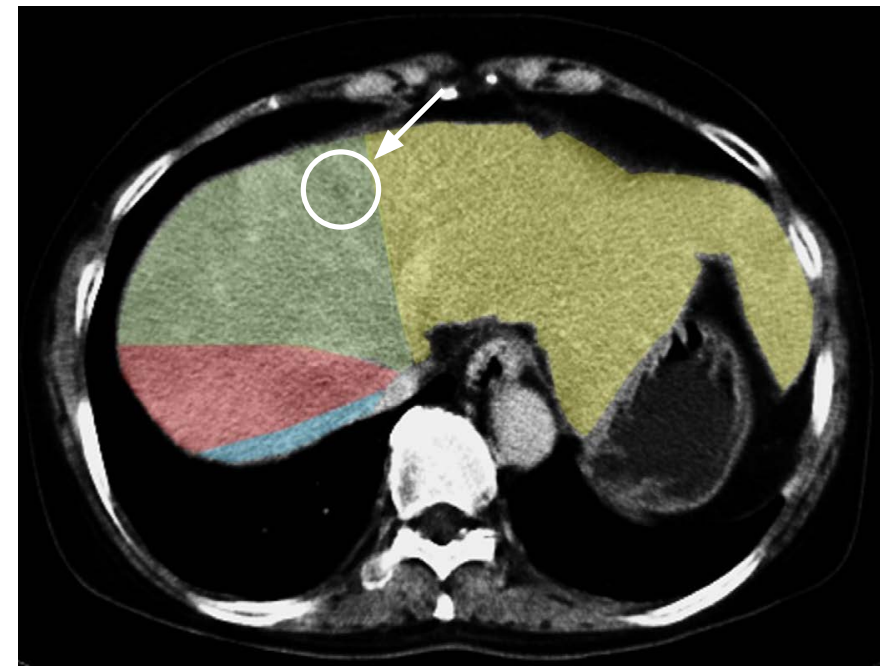

B

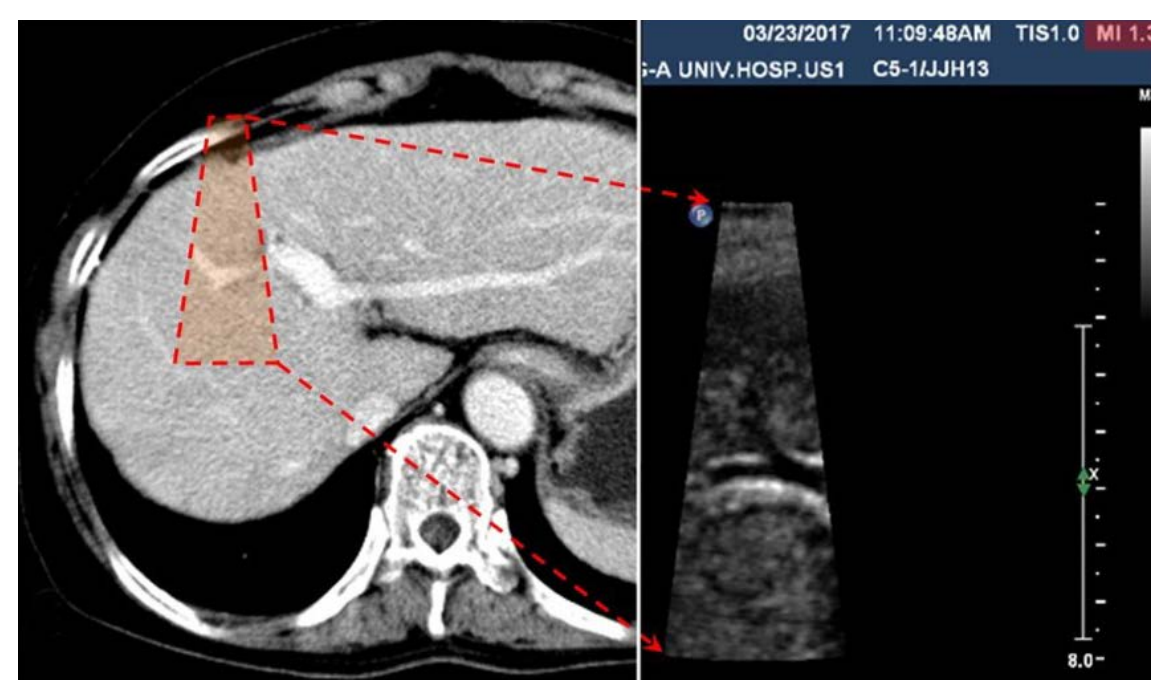

C

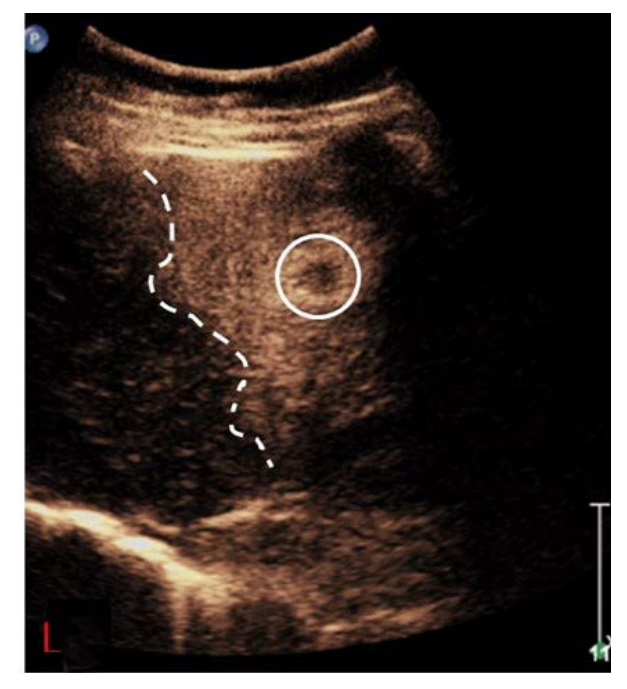

D

Fig. 5. Hepatocellular carcinoma in a 60-year-old woman with liver cirrhosis.

A, B. Dynamic enhanced computed tomography scans are shown. In the arterial phase (A), a small nodular lesion shows intense enhancement (circle and arrow). In the delayed phase (B), the nodule shows wash-out (circle and arrow). A hepatocellular carcinoma is located in S4 (blue area, S7; red area, S8; green area, S4; yellow area, S2). C. The target (S4) portal vein (red area with dashed line) is selected. After injection with Sonazoid, a high-mechanical index (MI) scan with a reduced field of view is applied. D. In the low-MI scan, S4 appears as a dark area with disruption of the Sonazoid (left side of the dashed line). S2 appears as a bright area with the Sonazoid in a static state (right side of the dashed line). The hepatocellular carcinoma is located in S2 (circle), not S4. The location was surgically confirmed to be $\mathbf{S}$, and a left lateral sectionectomy was performed.

locations of the masses in patients who did not undergo surgery with the Glissonean pedicle approach could not be confirmed. Additionally, the number of patients in this study was small. Therefore, further research with more patients is needed. Second, results may differ depending on the locations of the masses. It was more difficult to distinguish segments in the right lobe than in the left lobe. We assume that the parallel direction of the right portal vein to the sonic beam and right-lobe atrophic changes in patients with advanced cirrhosis were responsible for this result. Third, failures or difficulties may occur depending on patients' conditions. For example, bowel invagination can obscure the path of the US beam. If a patient breathes unevenly or is unable to stand for at least 5 minutes, it is difficult to target the specific portal vein long enough to destroy the Sonazoid microbubbles. Therefore, CEUS itself may fail in these situations.

We have suggested a new protocol for differentiating hepatic 
liver segments using CEUS with Sonazoid. The results of this study show that our novel high-and-low-MI CEUS protocol with Sonazoid helped preoperatively distinguish hepatic segments using portal vein flow, as in the Glissonean pedicle approach, thereby providing a less invasive way of acquiring the needed information.

ORCID: Taehyuk Ham: https://orcid.org/0000-0002-3432-2177; Joo Hee Jeon: https://orcid.org/0000-0002-4012-8338; Younghoon Roh: https://orcid.org/00000002-0165-0318; Sungwook Lee: https://orcid.org/0000-0001-9243-8488; Sangyoon Lee: https://orcid.org/0000-0001-9278-0312; Heejin Kwon: https://orcid.org/00000002-2001-953X; Jinhan Cho: https://orcid.org/0000-0002-5929-9399

\section{Author Contributions}

Conceptualization: Cho J, Kwon H, Roh Y, Lee Sungwook. Data acquisition: Roh Y, Lee Sungwook, Lee Sangyoon, Kwon H, Cho J. Data analysis or interpretation: Ham T, Cho J, Lee Sangyoon. Drafting of the manuscript: Ham T, Jeon JH, Lee Sangyoon. Critical revision of the manuscript: Cho J. Approval of the final version of the manuscript: all authors.

\section{Conflict of Interest}

No potential conflict of interest relevant to this article was reported.

\section{Acknowledgments}

This study was supported by research funds from Dong-A University.

\section{References}

1. Mullin EJ, Metcalfe MS, Maddern GJ. How much liver resection is too much? Am J Surg 2005;190:87-97.

2. Smith D, Downey D, Spouge A, Soney S. Sonographic demonstration of Couinaud's liver segments. J Ultrasound Med 1998;17:375-381.
3. Germain T, Favelier S, Cercueil JP, Denys A, Krause D, Guiu B. Liver segmentation: practical tips. Diagn Interv Imaging 2014;95:10031016.

4. Yamamoto M, Katagiri S, Ariizumi S, Kotera Y, Takahashi Y. Glissonean pedicle transection method for liver surgery (with video). J Hepatobiliary Pancreat Sci 2012;19:3-8.

5. Aragon RJ, Solomon NL. Techniques of hepatic resection. J Gastrointest Oncol 2012;3:28-40.

6. Majno P, Mentha G, Toso C, Morel P, Peitgen HO, Fasel JH. Anatomy of the liver: an outline with three levels of complexity: a further step towards tailored territorial liver resections. J Hepatol 2014;60:654662.

7. Jang JY, Kim MY, Jeong SW, Kim TY, Kim SU, Lee SH, et al. Current consensus and guidelines of contrast enhanced ultrasound for the characterization of focal liver lesions. Clin Mol Hepatol 2013;19:116.

8. Chung YE, Kim KW. Contrast-enhanced ultrasonography: advance and current status in abdominal imaging. Ultrasonography 2015;34:3-18.

9. Piscaglia F, Lencioni $R$, Sagrini $E$, Pina $C D$, Cioni $D$, Vidili $G$, et al. Characterization of focal liver lesions with contrast-enhanced ultrasound. Ultrasound Med Biol 2010;36:531-550.

10. Numata K, Luo W, Morimoto M, Kondo M, Kunishi Y, Sasaki T, et al. Contrast enhanced ultrasound of hepatocellular carcinoma. World J Radiol 2010;2:68-82.

11. Luo W, Numata $K$, Kondo M, Morimoto $M$, Sugimori K, Hirasawa $K$, et al. Sonazoid-enhanced ultrasonography for evaluation of the enhancement patterns of focal liver tumors in the late phase by intermittent imaging with a high mechanical index. J Ultrasound Med 2009;28:439-448.

12. Chouillard EK, Gumbs AA, Cherqui D. Vascular clamping in liver surgery: physiology, indications and techniques. Ann Surg Innov Res 2010;4:2. 